\title{
Research on the control technology of self-synchronous voltage source inverter for distributed parallel system
}

\author{
Zhong $\mathrm{Xu}^{1 *}$, Zhu Runqiu ${ }^{1}$, Hou Kai ${ }^{1}$, and Yuan Weifeng ${ }^{1}$ \\ ${ }^{1}$ NARI Technology Co., Ltd. , Nanjing 211106, Jiangsu Province, China
}

\begin{abstract}
In this paper, a small signal model based on droop controller is established for the selfsynchronous voltage source parallel system. The influence of droop coefficient on system stability is analysed, and the constraint conditions of droop coefficient are given. Based on the analysis of the parallel system of self-synchronous voltage source inverter, a multi-machines parallel control scheme of selfsynchronous voltage source inverter is formulated. The model and test platform are built to carry out the parallel simulation and experimental verification of distributed line impedance parameters. Simulation and experimental results show that the proposed control strategy can achieve good dynamic and steady-state power sharing.
\end{abstract}

\section{Introduction}

For the parallel system of distributed inverters, the connection points are randomly distributed in the distribution network, and the existing technology can not solve the active power and reactive power decoupling problem of voltage source inverter caused by the equivalent parameters of line impedance. With the increasing capacity of microgrid, it is urgent to solve the problem of distributed multi-machines parallel connection of self-synchronous voltage source inverter (SVI) to provide voltage and frequency support for distribution network.

In [1-2], the research on parallel system connection of multi-machines was carried out by using virtual synchronous generator (VSG). Although the scheme realizes simple droop control characteristics, but it is easy to cause power oscillation and belongs to differential regulation. In [3], the power decoupling matrix is added to the droop control loop to realize the accurate control of the inverter output power. However, the output impedance characteristics and line impedance characteristics of the inverter are not taken into account in this scheme, which is easy to cause circulating current in distributed multi-machines parallel system. In [4-6], an integral link and a first-order inertia link are added to the droop control of the inverter to realize the zero difference regulation of frequency and voltage of the multi-machines parallel system. However, the improved droop control increases the transient response time of the system, which is easy to cause power oscillation and voltage resonance in parallel.

In this paper, the parallel system of the SVI is taken as the research object. Based on the research of reference [7-8], the inner loop of current control, secondary voltage and frequency regulation control are introduced, and the distributed parallel networking technology is proposed to realize the stable operation of distributed multi-machines parallel system.

\section{Distributed multi-machines parallel system}

\subsection{Stability analysis of parallel system}

The output equivalent circuit of the SVI parallel system is shown in Fig.1. The small signal model of the system loop based on droop controller is established, and the influence of droop coefficient on the system stability is analysed.

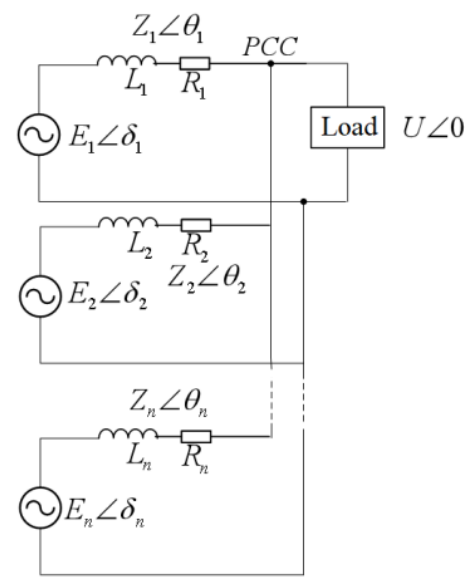

Fig. 1. Schematic diagram of parallel system

Where $E_{n} \angle \delta_{n}$ is the output voltage of the SVI, $U \angle 0$ is the AC bus voltage, $Z_{n} \angle \theta_{n}$ is the connection impedance of the SVI, which can be expressed as $Z_{n} \angle \theta_{n}=R_{n}+j \omega L_{n}=R_{n}+j X_{n}$.

\footnotetext{
* Corresponding author: zhongxu1989@163.com
} 
Taking the stability of the first SVI droop system as an example, the output active power and reactive power of SVI can be obtained from Fig. 1 as follows:

$$
\begin{aligned}
& P_{1}=\frac{U}{R_{1}^{2}+X_{1}^{2}}\left[R_{1}\left(E_{1} \cos \delta_{1}-U\right)+X_{1} E_{1} \sin \delta_{1}\right] \\
& Q_{1}=\frac{U}{R_{1}^{2}+X_{1}^{2}}\left[X_{1}\left(E_{1} \cos \delta_{1}-U\right)-R_{1} E_{1} \sin \delta_{1}\right]
\end{aligned}
$$

The small signal model based on the time-varying phasor theory is established. Substituting $R_{1}=r_{1}+L_{1} s$, $X_{1}=\omega L_{1}$ into expression of (1) and (2). Because of the three-phase symmetry of the SVI, its power can be equivalent to the sum of the power of three single-phase inverters, so the output active power and reactive power of three-phase inverter can be obtained as:

$$
\begin{gathered}
P_{1}=3 \frac{L_{1} s+r_{1}}{\left(L_{1} s+r_{1}\right)^{2}+\left(X_{1}\right)^{2}}\left(E_{1}^{2}-E_{1} U \cos \delta_{1}\right)+3 \frac{X_{1}}{\left(L_{1} s+r_{1}\right)^{2}+\left(X_{1}\right)^{2}} E_{1} U \sin \delta_{1} \\
Q_{1}=3 \frac{X_{1}}{\left(L_{1} s+r_{1}\right)^{2}+\left(X_{1}\right)^{2}}\left(E_{1}^{2}-E_{1} U \cos \delta_{1}\right)-3 \frac{L_{1} s+r_{1}}{\left(L_{1} s+r_{1}\right)^{2}+\left(X_{1}\right)^{2}} E_{1} U \sin \delta_{1}
\end{gathered}
$$

Where $E_{1} \approx U, \cos \delta_{1} \approx 1, \sin \delta_{1} \approx \delta_{1} \approx 0, D_{1}=\left(r_{1}+s L_{1}\right)^{2}+X_{1}^{2}$

By linearizing equations (3) and (4) at the steadystate operating point of the system, we can obtain as follows,

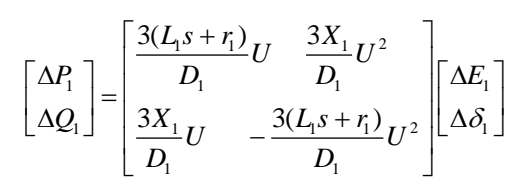

When the output line of the inverter is inductive, that is, the active power is only related to the angular frequency and the reactive power is only related to the amplitude, thus (5) can be simplified as follows:

$$
\left\{\begin{array}{l}
\Delta P_{1}=\frac{3 X_{1}}{D_{1}} U^{2} \Delta \delta_{1} \\
\Delta Q_{1}=\frac{3 X_{1}}{D_{1}} U \Delta E_{1}
\end{array}\right.
$$

\subsection{Stability analysis of active power and frequency droop system}

According to equation (6), the small signal equations of active power frequency and reactive power voltage droop can be obtained.

$$
\begin{aligned}
& \Delta \omega_{1}=-k_{p 1} \Delta P_{1} \\
& \Delta E_{1}=-k_{q 1} \Delta Q_{1}
\end{aligned}
$$

When calculating the active power and reactive power, a low-pass filter is usually added to filter of the active and reactive power, so as to get the average value of power as follows:

$$
\begin{gathered}
\Delta p_{1}=\frac{\omega_{f}}{s+\omega_{f}} \Delta P_{1} \\
\Delta q_{1}=\frac{\omega_{f}}{s+\omega_{f}} \Delta Q_{1}
\end{gathered}
$$

The active power and frequency droop loop diagram can be obtained from equation (8), which is shown in Fig. 2.

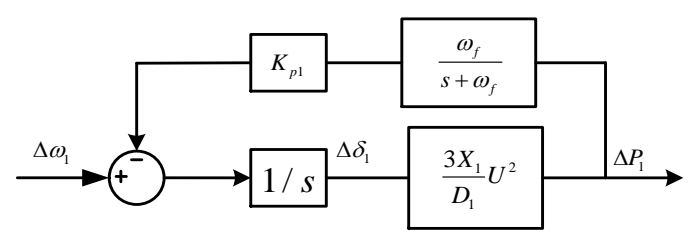

Fig. 2. Active power and frequency droop loop diagram

From Fig.2, the closed-loop transfer function of active frequency droop loop can be obtained as follows:

$$
\frac{\Delta P_{1}}{\Delta \omega_{1}}=\frac{3 X_{1} U^{2}}{s \cdot D_{1}+K_{p 1} \cdot 3 X_{1} U^{2} G(s)}
$$

In order to intuitively see the influence of droop coefficient $K_{p 1}$ on the stability of active power frequency droop loop, combined with the main circuit parameters of the SVI ( $L_{1}=0.15 \mathrm{~m} H, R_{1}=0.01 \Omega, C_{1}=600 \mu \mathrm{F}$ ), the root locus of equation (10) can be drawn with the change of droop coefficient $K_{p 1}$ as shown in Fig. 3 .

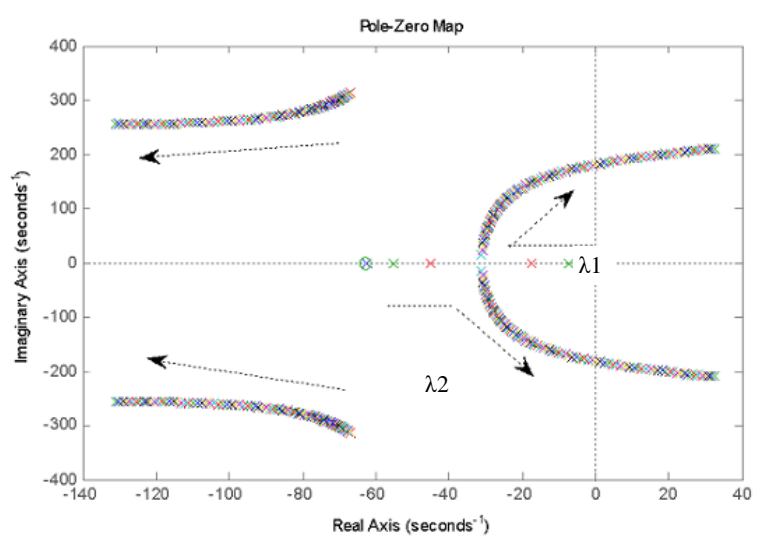

Fig. 3 (a) Root locus of active power And frequency droop closed loop transfer function

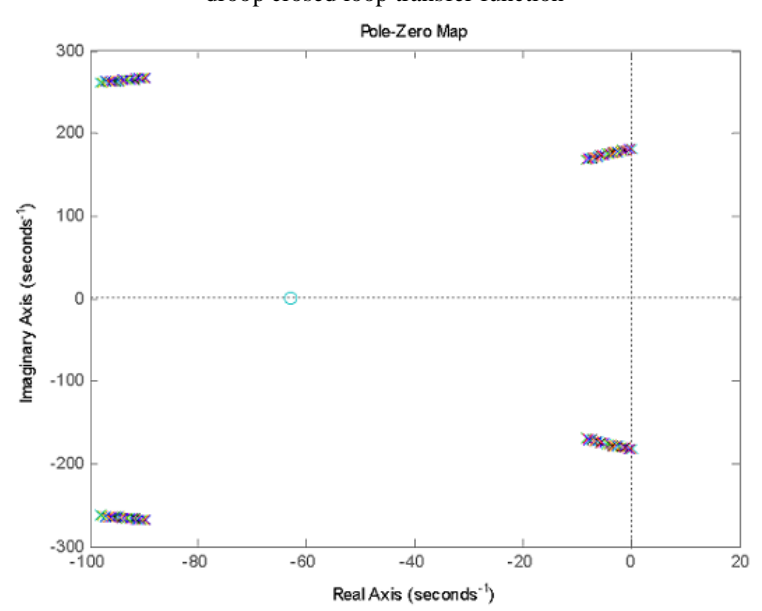

Fig. 3 (b) Critical stable root locus of active power frequency droop closed loop transfer function

Fig. 3. Stability analysis of active power and frequency droop system

It can be seen from Fig. 3 (a) that, $K_{p 1}$ within a certain value range (1e-7 1e-3), with the increase of $K_{p 1}$, the characteristic roots $\lambda_{1}$ and $\lambda_{2}$ move from the real root to the conjugate complex root, and the regulation oscillation period increases. At the same time, the conjugate complex root approaches the imaginary axis, the system damping decreases and the overshoot 
increases. When it exceeds a certain range, $\lambda_{1}$ and $\lambda_{2}$ will be located in the right half plane of $s$ domain, and the system will be unstable. Simulation results show that $K_{p 1}=6.8 \mathrm{e}-5$ is the critical point of system stability, and the root locus is shown in Fig. 3 (b).

\subsection{Stability analysis of reactive power and voltage droop system}

Similar to the stability analysis of active frequency droop loop, the system loop diagram of reactive power voltage amplitude droop can be obtained, as shown in Fig. 4.

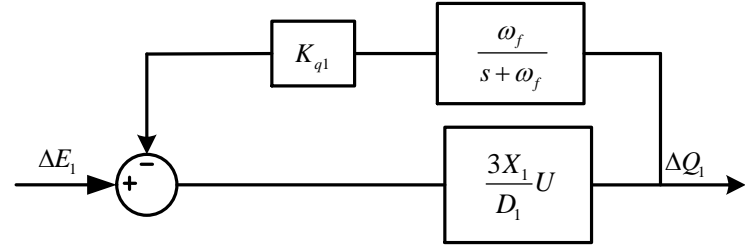

Fig. 4. System loop diagram of reactive power voltage droop

According to Fig. 4, the closed-loop transfer function of reactive power voltage amplitude droop loop can be obtained as follows:

$$
\frac{\Delta Q_{1}}{\Delta E_{1}}=\frac{3 X_{1} U}{D_{1}+K_{q 1} \cdot 3 X_{1} U G(s)}
$$

In order to intuitively see the influence of droop coefficient $K_{q 1}$ on the stability of reactive power voltage amplitude droop loop, it can be drawn according to formula (11). With the change of $K_{q 1}$ (1e-7 1e-3), the root locus of equation (11) is shown in Fig. 5.

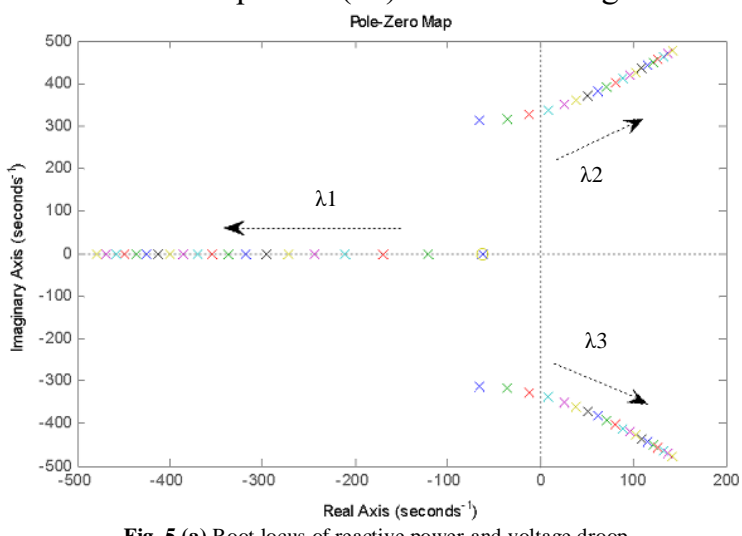

Fig. 5 (a) Root locus of reactive power and voltage droop closed loop transfer function

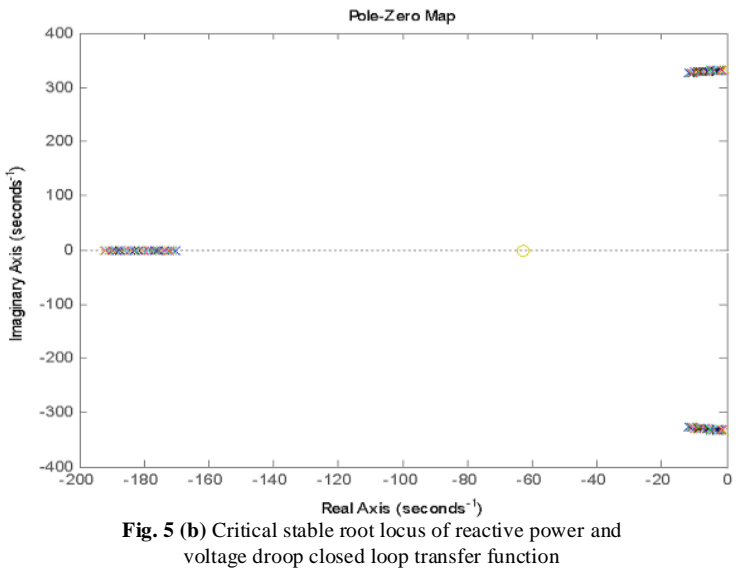

Fig. 5. Stability analysis of reactive power and voltage droop system

From the root locus diagram shown in Fig. 5 (a), it can be seen that when $K_{q 1}$ within a certain range of values, all three characteristic roots of the system are located in the left half plane of the s domain, and the system is in a stable state. At the same time, the characteristic roots $\lambda_{2}$ and $\lambda_{3}$ are conjugate complex roots, moving away from the real axis, the oscillation of the system regulation process increases. But when the droop coefficient continues to increase beyond a certain range, the two eigenvalues $\lambda_{2}$ and $\lambda_{3}$ of the system will be located in the right half plane of $\mathrm{s}$ domain, and the system will be unstable. The simulation results show that $K_{q 1}=1.26 \mathrm{e}-4$ is the critical point of system stability, and the root locus is shown in Fig. 5 (b).

According to the small signal modeling and stability analysis of SVI active power frequency and reactive power voltage loops in Fig. 3 (a) and Fig. 5 (a), $K_{p 1}$ and $K_{q 1}$ should be set within a certain range, otherwise the closed loop eigenvalue of simulation loop will move to the right half plane of s domain, resulting in system instability.

\subsection{Distributed parallel network control scheme}

The proposed control strategy of the SVI is shown in Fig. 6. For the multi-machines parallel system of the SVI, due to the unified control strategy, each inverter has the same characteristics of frequency and active power droop, voltage and reactive power droop. Therefore, when the parallel system is running, it has the natural dynamic and steady-state power sharing technology advantages.

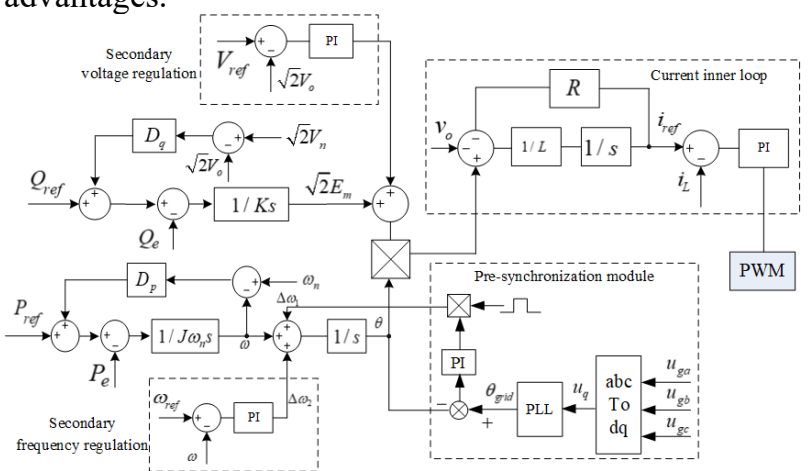

Fig. 6. Control block diagram of the SVI

Considering that the load of the SVI has certain requirements for voltage stability or voltage accuracy when it works in off grid state, in order to improve the output voltage and frequency accuracy, current inner loop, secondary voltage regulation and frequency regulation are introduced into the control strategy.

For the SVI, when switching from off grid to grid connected, in order to reduce the system current and voltage impact at the moment of grid connection of SVI, it is still necessary to introduce grid pre-synchronization link into the existing control strategy.

Fig. 6 shows that when the SVI receives the grid connected enabling signal, the SVI obtains the grid 
voltage phase $\theta_{\text {grid }}$ through the phase lock, and generates $\Delta \omega_{1}$ through the pre-synchronization module. Adjusting the phase angle $\theta$ of the SVI by $\Delta \omega_{1}$, the phase pre-synchronization process of the SVI is completed in the final tracking. After that, the grid connected switch is closed to realize the switch from off grid to grid connected.

\section{Simulation analysis and experimental verification}

\subsection{Simulation analysis}

In order to verify the correctness of the control strategy for the multi-machines parallel system of the SVI, four SVIs parallel system model are built in PLECS. The parameters of four SVIs are the same.

In the case of off grid, four SVIs are connected to the grid in turn at $0.8 \mathrm{~s}, 1.5 \mathrm{~s}, 2.2 \mathrm{~s}, 3 \mathrm{~s}$. The output current waveform of the SVIs is shown in Fig.7(a), the waveform of load voltage is shown in Fig.7(b), and the output active power is shown in Fig.7(c).

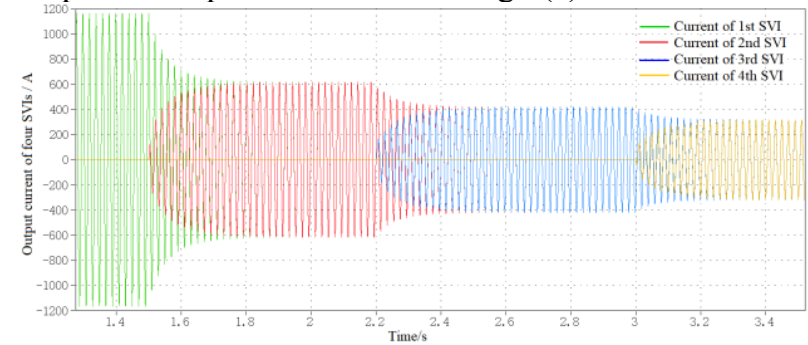

Fig. 7 (a). Output current waveform of SVI

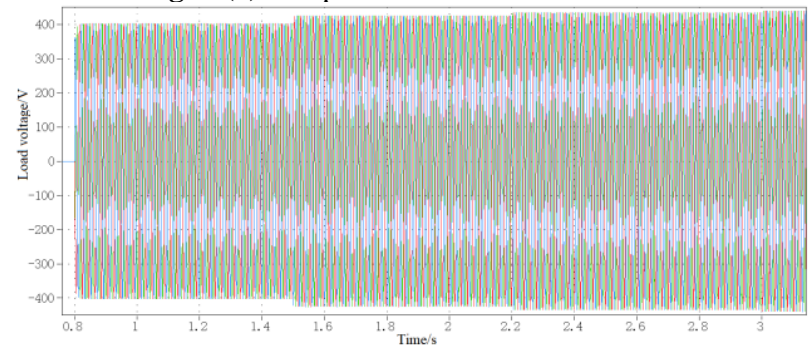

Fig. 7 (b). Waveform of load voltage

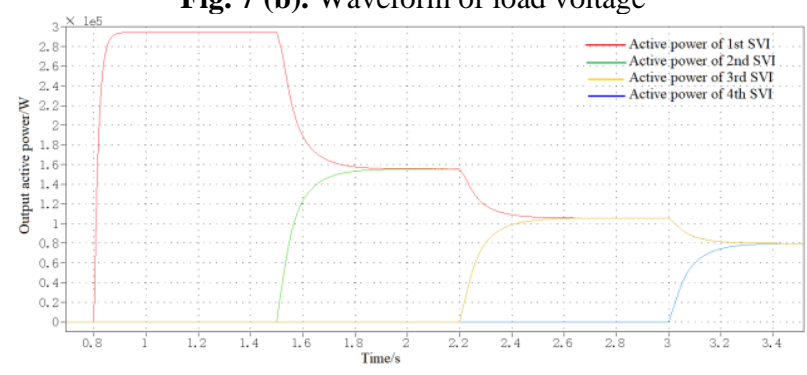

Fig. 7 (c). Output active power waveform of SVI

From the simulation waveform, it can be seen that the first SVI supplies power to the load at $0.8 \mathrm{~s}$, and the load voltage decreases due to the droop effect. At the time of $1.5 \mathrm{~s}$, the second SVI supplies power to the load at the same time, the droop effect is reduced, and the load voltage is increased. At $2.2 \mathrm{~s}$ time, the third SVI delivers power to the load, and the power is evenly distributed. At $3 \mathrm{~s}$ time, the fourth SVI supplies power to the load, and the power is divided equally. The impulse current is very small in the whole switching process, and a good smooth transition is achieved.

In the case of grid connection, four SVIs are connected to the grid at the same time and the given output active power is $50 \mathrm{~kW}, 100 \mathrm{~kW}, 200 \mathrm{~kW}$ and $300 \mathrm{~kW}$ respectively. At the beginning, the four SVIs are off grid and the grid supplies power to the load. At $0.8 \mathrm{~s}$, four SVIs are connected to the grid, and the output current waveform of the inverter is shown in Fig. 8 (a), the waveform of load voltage is shown in Fig. 8 (b), and the output current of phase a between the grid and the 50kW SVI is shown in Fig. 8 (c),

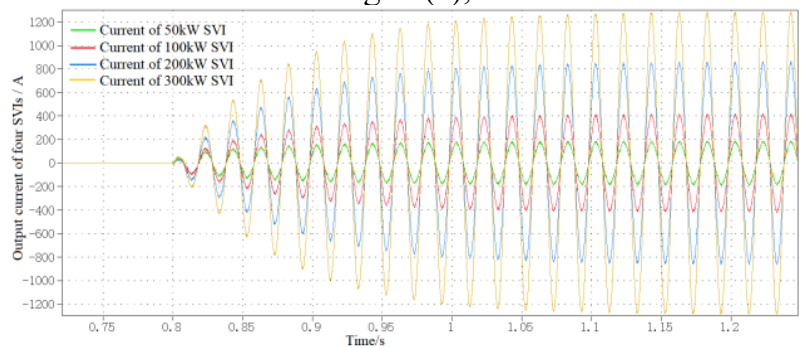

Fig.8 (a). Output current waveform of SVI

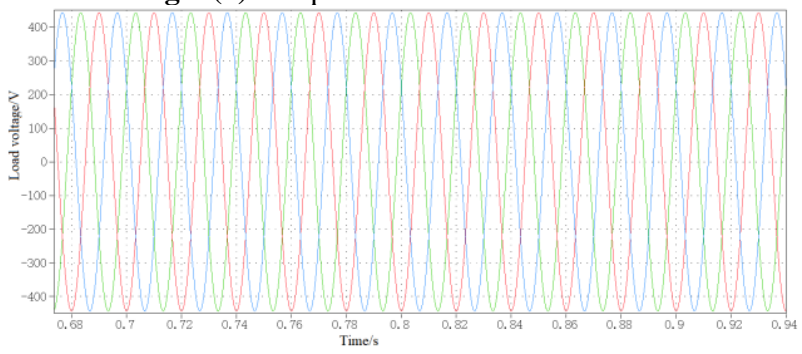

Fig. 8 (b). Waveform of load voltage

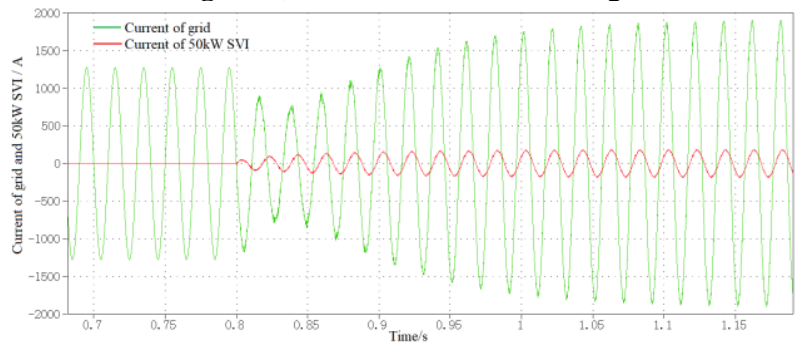

Fig.8 (c). Output current waveform of grid and 50kW SVI

The simulation waveform shows that when the four SVIs given different power, they supply power to the load according to their given power. Since the total output power of the four SVIs is greater than the load power, the grid absorbs the active power. In the process of the SVI from off grid to grid connected, the impulse current is very small, and a good smooth transition is achieved. At the same time, due to the clamp of the power grid, the load voltage remains unchanged.

\subsection{Experimental verification}

In order to verify the steady-state and dynamic characteristics of the parallel system with multiple SVIs under different load conditions, a four-machine parallel 
system is built in the test platform to carry out the fourmachines parallel test, and the single SVI is set at $500 \mathrm{~kW}$. The main circuit parameters of the SVI are the same as those of simulation.

Under full load, the output current and voltage waveforms of the four parallel SVIs are shown in Fig. 9. It can be seen from the experimental waveforms that when the four SVIs operate in parallel, the load power is well distributed, and the excellent steady-state current sharing characteristics are achieved.

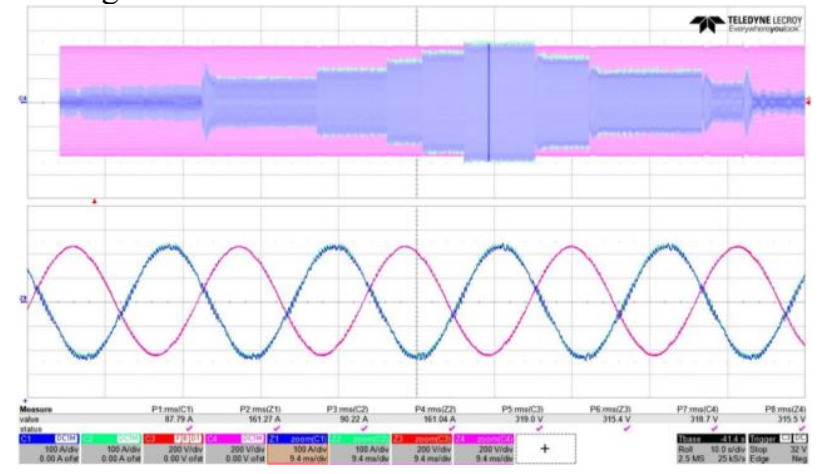

Fig. 9 (a). Current and voltage waveform at full load

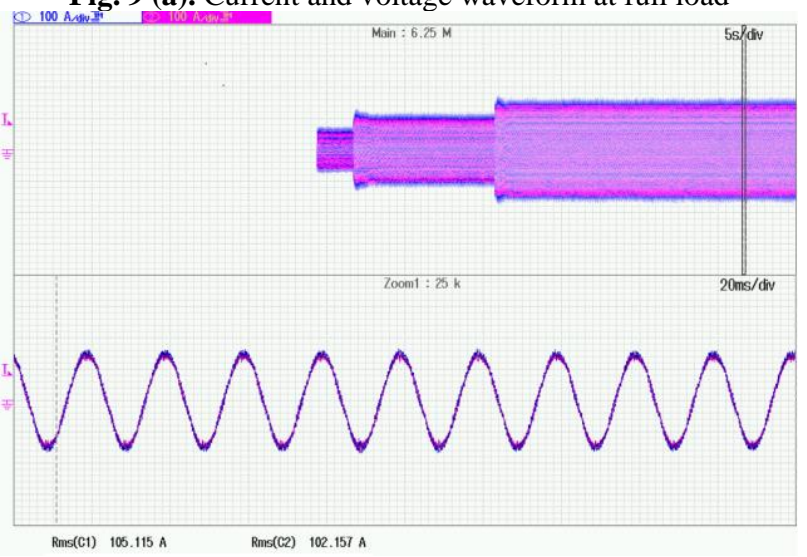

Fig. 9 (b). Steady state current waveform at other power stages

\section{Conclusion}

In this paper, a small signal model based on droop controller is established for the parallel system. The influence of droop coefficient on system stability is analysed, and the constraint conditions of droop coefficient are given. In order to improve the accuracy of output voltage and reduce the impact of grid connection on system current and voltage, secondary voltage regulation module, frequency modulation module and grid pre-synchronization module are introduced into the control strategy. Based on the analysis of parallel system, a distributed multi-machines parallel control scheme of the SVI is developed. A four machine distributed parallel simulation model and test platform are built to carry out the multi-machines parallel experiment verification under distributed line impedance parameters. The simulation and experimental results show that under the proposed control strategy, the four SVIs have good dynamic regulation ability and steady-state characteristics when the four machines parallel operation of the SVI. The dynamic, steady-state active and reactive power sharing is well realized, and the multi-machines parallel network of the SVI is realized Stable operation of the system.

\section{References}

1. Q C Zhong. Robust Droop Controller for Accurate Proportional Load Sharing Among Inverters Operated in Parallel. IEEE Transactions on Industrial Electronics, 60(4): 1281-1290. (2013)

2. Q C Zhong, Y Zeng. Parallel Operation of Inverters with Different Types of Output Impedance. Industrial Electronics Society, IECON 2013-39th Annual Conference of the IEEE, Vienna, 1398-1403. (2013)

3. Y Li, Y W Li. Decoupled Power Control for An Inverter Based Low Voltage Microgrid in Autonomous Operation. IEEE 6th International Power Electronics and Motion Control Conference (IPECM 2009) pp 2490-2496. (2009)

4. K. De Brabandere, B. Bolsens, J. Van den Keybus, et al. A Voltage and Frequency Droop Control Method for Parallel Inverters. IEEE Transactions on Power Electronics, 22(4): 1107-1115. (2007)

5. Josep M. Guerrero, Luis García de Vicuňa, José Matas, et al. Output Impedance Design of ParallelConnected UPS Inverters with Wireless LoadSharing Control. IEEE Transactions on Power Electronics, 52(4): 1126-1135. (2005)

6. CHANDORKAR M C, DIVAN D M, ADAPA R. Control of parallel connected inverters in standalone ac supply systems. IEEE Trans on Industry Applications, 29(1): 136-143. (1993)

7. Q H Ji, Z Wei, et al. Research on control strategies of self-synchronous voltage inverter parallel system. Power Electronics. 51(4): 46-49. (2017)

8. X Q Ru, Z Wei, et al. Research on control strategy of self-synchronous voltage source inverter. Power Electronics. 50(12): 84-87. (2016) 